Questions vives

\section{Questions Vives}

Recherches en éducation

Vol.7 $n^{\circ} 17$ | 2012

Des usages des TIC à la certification des compétences numériques : quels processus de formation et de validation?

\title{
Implantation du C2i niveau 1 au sein de l'école normale supérieure de Marrakech
}

\section{Somaya el Gharras et Lahoussaine Ait Ounejjar}

\section{(c) OpenEdition}

Édition électronique

URL : http://journals.openedition.org/questionsvives/1047

DOI : 10.4000 /questionsvives. 1047

ISBN : 978-2-8218-1394-6

ISSN : $1775-433 \mathrm{X}$

Éditeur

Université Aix-Marseille (AMU)

Édition imprimée

Pagination : 147-155

ISSN : 1635-4079

Référence électronique

Somaya el Gharras et Lahoussaine Ait Ounejjar, «Implantation du C2i niveau 1 au sein de l'école normale supérieure de Marrakech », Questions Vives [En ligne], Vol.7 n¹7 | 2012, mis en ligne le 15 novembre 2012, consulté le 03 mai 2019. URL : http://journals.openedition.org/questionsvives/1047 ; DOI : 10.4000/questionsvives. 1047

Ce document a été généré automatiquement le 3 mai 2019.

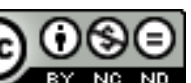

Questions Vives est mis à disposition selon les termes de la licence Creative Commons Attribution Pas d'Utilisation Commerciale - Pas de Modification 4.0 International. 


\title{
Implantation du C2i niveau 1 au sein de l'école normale supérieure de Marrakech
}

\author{
Somaya el Gharras et Lahoussaine Ait Ounejjar
}

\section{Témoignage}

1 Le projet global d'implantation du C2i dans les universités marocaines vise la formation des cadres supérieurs et en particulier, ceux qui pilotent des dispositifs d'éducation, d'enseignement et de formation développant et/ou utilisant les TIC. Le personnel d'encadrement des établissements universitaires au Maroc est aujourd'hui, pour une très large part, autodidacte du domaine des TIC puisque, à ce jour, aucune formation académique n'est mise en place.

2 La démarche suivie pour l'implantation du C2i est descendante: formation des formateurs, puis formation initiale des futurs enseignants du secondaire, puis diffusion du savoir dans les cycles secondaires.

\section{Contexte}

3 L'École Normale Supérieure de Marrakech (ENS) est un établissement étatique de formation des enseignants du secondaire (lycée : élèves de 15-18 ans). Dans ce contexte, l'informatique est enseignée depuis de nombreuses années aux étudiants de toutes les filières hébergées dans notre institution. Le contenu de cette discipline reste à l'initiative de l'enseignant qui l'assure. Selon les sections, ce cours est de l'ordre de l'initiation à l'utilisation de l'ordinateur tout comme il peut être autour des applications pédagogiques de l'ordinateur dans la discipline. 
4 Le développement de l'usage de l'outil informatique dans le milieu scolaire marocain à l'initiative du ministère de l'éducation via le programme Génie $^{1}$ qui a pour objectif d'équiper, de connecter à l'Internet tous les établissements scolaires (primaires, collèges et lycées) puis de former des personnes ressources en informatique et enfin de produire des contenus numériques destinés à l'enseignement secondaire, nous a amené à réfléchir sur la formation dispensée à nos étudiants qui seront des acteurs futurs pour la réussite de ce projet. Nous avons alors pris l'initiative de chercher à unifier le contenu de cet enseignement en vue de faire en sorte que tous les futurs lauréats de notre établissement puissent acquérir des compétences basiques en technologie de l'information et de la communication pour l'enseignement (TICE). Cette initiative se trouve en adéquation avec l'esprit de la certification C2i (Certificat Informatique et Internet instauré dans les universités françaises). Toutefois, elle reste ponctuelle et n'est pas soutenue par un cadre juridique cohérent (absence de circulaire ministérielle institutionnalisant la certification).

5 L'ENS est intégrée l'université Cadi Ayyad de Marrakech qui, elle-même, est consciente de l'impact positif que pourrait avoir une uniformisation du contenu enseigné et exigé de ses étudiants en matière de TICE et qui, dans ce sens, a signé une convention de partenariat avec une université française pour la certification C2i1 des étudiants a jugé important de se greffer à ce projet. Un comité de pilotage intégrant les instances des différents établissements de l'université a été mis en place pour assurer le suivi de l'implantation de la certification.

6 Rappelons que les étudiants lauréats du concours d'accès aux ENS au Maroc disposent minimalement d'une licence (Baccalauréat +3 ans d'études universitaires). Leur niveau en termes d'usages des TIC est très hétérogène du fait du manque d'uniformité dans la formation en ce domaine. Quelques universités marocaines en sont à leur début dans l'expérience d'implantation du C2i1 dans le cursus universitaire. Pour le moment, cette expérience se déroule au niveau master et/ou doctorat. Par conséquent, le lauréat de la licence n'est pas encore concerné. Ce qui justifie notre initiative d'intervention à ce niveau de la formation.

7 Par ailleurs, la vocation des ENS étant la formation des enseignants, il nous a semblé important de cibler par cette action aussi bien la formation initiale de l'étudiant que l'uniformisation des compétences en ce domaine du personnel éducatif de notre établissement. Nous avons jugé utile de faire en sorte que l'enseignant, par sa propre certification, soit en mesure de juger et d'exploiter les compétences de ses étudiants en la matière.

\section{Stratégie d'implantation}

8 À partir des conditions décrites ci-dessus et tenant compte des spécificités du contexte, nous avons défini un plan d'action de mise en œuvre du projet d'implantation du C2i au sein de l'ENS. Dans le présent travail, nous nous limiterons à l'expérience menée au sein de l'ENS de Marrakech qui s'intègre toutefois dans le cadre institutionnel de l'université 
de rattache (UCA). Dans une première étape de notre expérience (2009-2011), le plan d'action retenu est le suivant :

- Organisation d'une journée d'étude au sein de l'établissement autour, d'une part, des technologies de l'information et de la communication à travers le programme GENIE et d'autre part de la certification C2i.

- Organisation de tests de positionnement C2i niveau1.

- Expérimentation et déploiement d'une plateforme locale d'autoformation pour le C2i niveau1 (C2i1)

- Formation sur l'utilisation d'une plateforme de formation à distance (Moodle).

- Planification de sessions de formation et d'appui pour la mise à niveau en vue de faciliter la certification.

- Participation active, avec le comité de pilotage, à des sessions de réflexion pour accélérer les modalités de généralisation de la certification au sein de notre université.

Ce plan d'action s'est déroulé en deux phases. Dans la première phase, nous avons jugé utile de cibler, en premier lieu, le personnel éducatif pour les raisons précitées. Ce public est constitué d'enseignants chercheurs et d'administratifs (tous enseignants chargés de tâches administratives ou de tâches de préparation d'expériences au sein des divers départements de l'établissement). La deuxième phase concerne les étudiants de l'établissement.

Un modèle de formation a été mis en place se déclinant en une étape "sensibilisation ", suivie d'une étape « formation » se terminant par l'étape « certification ».

Il en découle un planning de formation couvrant aussi bien la partie théorique que pratique des neufs domaines du référentiel $\mathrm{C} 2 \mathrm{i} 1^{2}$. Parallèlement, un ensemble de ressources (cours, présentation assistée par ordinateur, Travaux pratiques, tests, url...) est mis à disposition des candidats sur la plateforme de l'ENS. Le cours théorique est, chaque fois, suivi d'un test d'évaluation des connaissances par domaine ${ }^{3}$. Des séances de type examen pratique C2i ont été menées par les coordinateurs du projet pour synthétiser l'état des connaissances des candidats potentiels.

\section{Phase I: Certification du personnel éducatif}

12 Au moment du lancement de l'opération, 33 participants ont adhéré à cette expérience, parmi lesquels 27 enseignants chercheurs et 6 administratifs. Suite à la journée d'étude, un questionnaire élaboré par nos soins a été distribué aux candidats potentiels. Il a été rempli et rendu par 14 enseignants chercheurs et un administratif. L'objectif de ce questionnaire est de laisser au candidat le soin d'auto évaluer ses compétences dans le domaine de ses propres usages de l'informatique et de l'Internet sur une échelle de 1 à 5. Étant donné que les personnes interrogées sont familiarisées avec des termes qui représentent, sans être exhaustifs, plus explicitement les domaines du référentiel C2i niveau1, les questions posées sont volontairement tirées du quotidien d'un usager de l'ordinateur.

Il ressort de cette enquête que les usages jugés maîtrisés de l'ordinateur sont par ordre décroissant :

1. La messagerie

2. Le système d'exploitation

3. Le traitement de texte 
4. La navigation et la recherche Internet

5. La présentation assistée par ordinateur. résultats représentés par le graphique suivant.

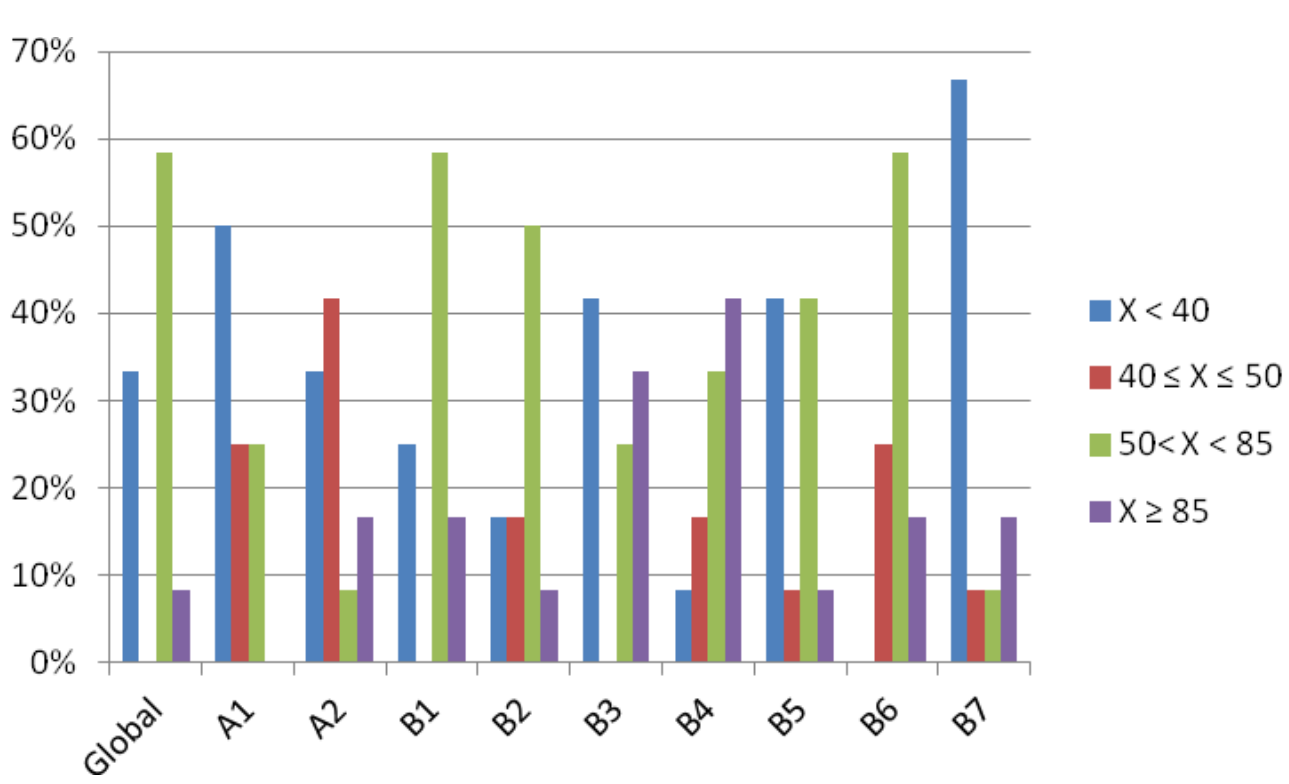
désigne le score obtenu par le candidat globalement puis dans chaque domaine de compétences. Nous avons donc mis en relief 4 catégories :

- Peu initiés $(\mathrm{X}<40 \%)$

- Initiés $(40 \% \leq \mathrm{X} \leq 50 \%)$

- Expérimentés $(50 \%<\mathrm{X}<85 \%)$

- Avancés $(X \geq 85 \%)$

En particulier, les catégories «Expérimentés » et "Avancés » semblent potentiellement certifiables (8 candidats soit $66,6 \%$ ) tandis que les catégories «Peu initiés » et "initiés » (4 candidats) auraient besoin de formation complémentaire. Ceci étant le résultat d'un premier test de positionnement donc non significatif de l'état des connaissances réelles des candidats, la formation mise en place est ouverte à l'ensemble des participants (33). 
18 Notons que les meilleurs scores obtenus concernent les domaines de compétences dans lesquels les candidats se sont auto-évalués positivement.

Premièrement :

- Environnement de travail

- Réaliser des documents destinés à être imprimés

- Échange et communication

Deuxièmement :

- Recherche de l'information

- Sécurité, sauvegarde et archivage

Troisièmement :

- Présentation assistée par ordinateur.

19 Une seconde analyse plus fine des résultats du test de positionnement a été réalisée en vue de déterminer les points les plus sensibles chez les personnes en formation. Cette identification des questions auxquelles les candidats n'ont pas répondu correctement a permis de reconnaître les besoins spécifiques par domaine des candidats pour établir un plan et un contenu de formation adaptés. L'accent a été mis, lors des formations dispensées, sur les savoirs non maîtrisés mis en évidence par l'analyse du test de positionnement.

20 La formation était ouverte aux 33 candidats potentiels. 30 candidats (24 enseignants et 6 administratifs) se sont présentés à la certification. Finalement, 18 personnes ont été certifiées. Le taux de réussite global est $60 \%$ (14 enseignants sur 24 et 4 administratifs sur 6).

\section{Phase II : Certification des étudiants}

21 Désormais, riche d'une équipe d'enseignants certifiés, l'expérience a été menée l'année suivante avec nos étudiants. Toutefois, étant données les ressources humaines et matérielles disponibles, nous avons dû limiter l'expérimentation aux sections scientifiques (mathématiques, Sciences physiques, sciences de la vie et de la terre, informatique). L'effectif total des bénéficiaires est de 174 candidats répartis en groupes.

\section{Résultats du test de positionnement}

22 Le graphique 2 suivant résume le pourcentage de candidats ayant obtenu un score $\mathrm{X}$ (score global, puis score par domaine) compris dans les intervalles précédemment définis. 
Graphique 2 : Illustration graphique des pourcentages de candidats ayant obtenu un score X.

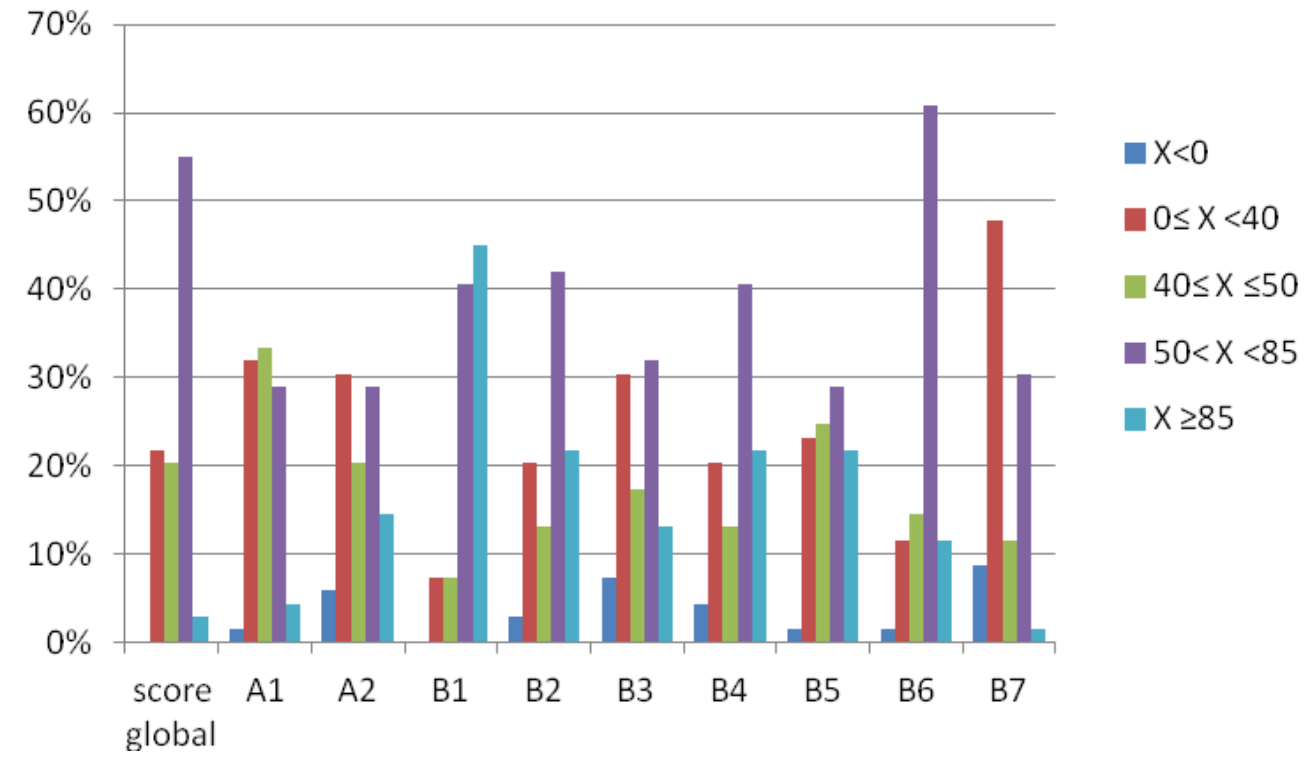

$58 \%$ des candidats se situent dans les catégories «Expérimentés » et "Avancés », $42 \%$ dans les catégories «Peu initiés» et "Initiés». Ces taux seraient-ils des indicateurs potentiels du taux de réussite à la certification?

Les domaines les mieux réussis par ordre décroissant sont : B1 (85,51\%), B6 (72,46\%), B2 (63,77\%), B4 (62,32 \%), B5 (50,72 \%).

Ces résultats s'expliquent, d'une part, par les enseignements d'informatique dispensés lors de leur scolarité antérieure (lycée et université) et, d'autre part, par leur usage courant des outils de communication (messagerie, forum, Web...). Notons que les outils destinés à produire des documents (traitement de texte, tableur, PAO) arrivent en seconde place. Les domaines les moins réussis sont : B7 (31,88 \%) et A1 (33,33\%).

Ces résultats montrent que les étudiants sont peu familiarisés avec les outils TICE de «travail collaboratif » et ont une connaissance limitée en ce qui concerne les statuts de logiciels (libre, freeware, shareware, logiciel du domaine public) ainsi que les formats de fichiers (image, son, vidéo...). Nous remarquons que les scores négatifs apparaissent chez ce public, chose que nous n'avions pas relevée lors de la première expérience. Nous pouvons expliquer ce phénomène par le fait que l'enseignant a une plus grande conscience des effets des réponses aléatoires sur un QCM.

172 candidats se sont présentés à la certification dont 22 ont été certifiés. Ce qui nous donne un taux de réussite global de $12,8 \%$. Y aurait-il une surestimation par le candidat de ses compétences réelles qui se justifie par son usage quotidien de l'ordinateur et pour certains (section informatique) par le fait que cela concerne sa spécialité ? En d'autres termes, ces derniers ne surestiment-ils pas la place du savoir dans la compétence?

\section{Bilan de l'expérimentation}

\section{Bilan phase I}

La certification initialement programmée en juin, donc juste après la formation, a dû être retardée pour des raisons purement administratives jusqu'en octobre de la même année. 
Cette période creuse entre la formation et la certification a générée d'une part un désengouement et d'autre part une perte d'efficacité de la formation. La certification se déroule en présentiel sous l'égide de l'université Cadi Ayyad et d'un correspondant français du C2i1. La période de certification coïncide avec la rentrée universitaire, période durant laquelle le personnel éducatif est largement sollicité pour les tâches de mise en place du cursus universitaire de l'année en cours.

Le public cible de cette phase étant initié mais aussi motivé par l'amélioration de ses compétences en matière de TICE, la formation a eu un impact positif sur l'appropriation des connaissances déjà en place et l'acquisition de nouveaux savoirs. Le fait de mettre le doigt sur les difficultés de chacun suite à l'analyse du test de positionnement a permis une remédiation personnalisée des difficultés de chacun des candidats. Le taux de réussite de cette catégorie est intéressant $(60 \%)$. Nous avons noté un intérêt pour le produit (C2i1) mais aussi pour l'approche de formation adoptée. Nous estimons que, sans la perte de candidats due aux raisons explicitées précédemment, le pourcentage de candidats certifiés aurait été plus important.

\section{Bilan phase II}

La formation et la certification C2i1 s'étant faite dans le cadre du module TICE entrant dans les exigences de leur formation, il n'y a quasiment pas eu de perte de candidats. Nous avons identifié les points suivants ayant pu influer sur le taux de réussite obtenu :

- Hétérogénéité du public. Certains candidats ont de grandes potentialités tandis que d'autres n'ont jamais eu accès à un ordinateur étant donné la diversité de leurs établissements d'origine, de leurs cursus et de l'année d'obtention de leur diplôme.

- Surestimation par le candidat de ses propres compétences.

- Utilisation inefficace des ressources mises à disposition du candidat sur les plateformes d'enseignement à distance (plateforme de l'UCAM et plateforme de l'ENS).

- Le statut secondaire du module TICE en regard des modules liés à la spécialité.

- Pour la section informatique, le contenu du programme C2i est faussement jugé facile, secondaire, acquis...

\section{Bilan global}

31 L'expérience menée au sein de l'ENS a donné un taux de réussite de $60 \%$ sur la catégorie «Enseignants/administratifs » et de $12,8 \%$ sur la catégorie "étudiants ». Ce qui nous donne un taux de réussite global de $19,8 \%$ pour cette première expérience. En France, actuellement, le taux de réussite global ${ }^{4}$ pour l'année $2009-2010$ est de $36 \%$. Notons que la première phase de certification (2004-2005) en France a donné lieu à un taux de réussite de $48,51 \%$.

\section{Stratégie de déploiement du C2i1}

L'expérience de l'ENS n'est pas un cas isolé au Maroc en général et à l'université Cadi Ayyad de Marrakech en particulier. Dans cette perspective, l'université envisage un déploiement de la certification C2i1 à plus grande échelle. Les enseignants de l'ENS étant intervenus dans les formations et ayant été certifiés ont développé des compétences et 
des savoir-faire permettant d'élargir le noyau initial de formateurs pour constituer une équipe complète.

Notre vision globale de l'avenir de cette expérience est :

- Formation et certification C2i1 en deuxième année de licence.

- Formation et certification C2i1 en première année de master pour les étudiants provenant d'autres structures.

- Formation et certification C2i2e enseignant en deuxième année de master.

- Formation et certification C2i1 pour les enseignants en poste dans le cadre de leur formation continue.

- Formation et certification C2i2e enseignant pour les enseignants en poste qui auraient été certifiés niveau1.

\section{Conclusion}

Dans le contexte actuel, la formation et la certification C2i1 concerne les étudiants inscrits en première année de master de toutes les filières ainsi que les enseignants et administratifs qui ne sont pas encore certifiés.

Les masters en cours à l'ENS sont des formations avec une charge de travail dans les disciplines de spécialité importante. La certification C2i nécessite un temps d'appropriation dont ne disposent pas les candidats sur la durée effective prévue pour la formation.

En conclusion, les résultats obtenus lors de cette expérimentation sont encourageants vu le contexte actuel (contraintes liées à la durée de la formation, absence d'institutionnalisation de la démarche ...) et en regard de l'expérience française.

\section{NOTES}

1. Programme génie (GÉNéralisation des Technologies d'Information et de Communication dans l'Enseignement au Maroc) : http://www.geniemaroc.com

2. Site officiel du c2i : http ://www.c2i.education.fr/

3. Site d'autoévaluation wims (université de Nice): http://wims.unice.fr/wims/wims.cgi? session=1H0E5C831D.2\&+lang=fr\&+module=local\%2Fdnte\%2FC2I-unice.fr

4. Résultats de l'observatoire du C2in1 : http://www.c2i.education.fr/spip.php?article93 


\section{RÉSUMÉS}

L'objectif de ce témoignage est de décrire l'expérience de l'implantation de la Certification Informatique et Internet niveau 1 (C2i1) vécue à l'École Normale Supérieure de l'université Cadi Ayyad de Marrakech. L'idée prépondérante est de faire en sorte que, dans un premier temps, le personnel enseignant et administratif en poste puisse identifier ses acquis, puis compléter ses connaissances pour enfin obtenir une certification attestant de ses compétences en ce domaine. La constitution d'une équipe de formateurs certifiés permettra ensuite d'agir au niveau de la formation initiale des étudiants qui seront des futurs enseignants. Nous décrirons l'approche adoptée pour le déroulement de l'expérience et les résultats obtenus. À l'issue de cette expérience, nous allons tenter d'identifier une stratégie de déploiement du C2i1 à une plus large envergure. L'approche adoptée a permis de diagnostiquer l'état des connaissances des acteurs de l'éducation, et de mettre en place une formation adaptée aux besoins du public cible.

\section{INDEX}

Mots-clés : certificat, C2i, formation, enseignant, TICE

\section{AUTEURS}

\section{SOMAYA EL GHARRAS}

Enseignant chercheur, École Normale Supérieure, université Cadi Ayyad Marrakech, Groupe de Recherche En Didactique de l'Informatique et des Mathématiques (GREDIM).

\section{LAHOUSSAINE AIT OUNEJJAR}

Enseignant chercheur, École Normale Supérieure, université Cadi Ayyad Marrakech, Groupe de Recherche En Didactique de l'Informatique et des Mathématiques (GREDIM). 